\title{
Antibiotics for the common cold: expectations of Germany's general population
}

M S Faber (faberm@rki.de)1,2, K Heckenbach ${ }^{1}$, E Velasco ${ }^{1,3}$, T Eckmanns $^{1}$

1. Department for Infectious Disease Epidemiology, Robert Koch Institute, Berlin, Germany

2. Postgraduate training for applied epidemiology (German Field epidemiology training programme), Robert Koch Institute, Berlin, Germany

3. Medical Faculty, Charité Universitätsmedizin, Berlin, Germany

Citation style for this article:

Faber MS, Heckenbach K, Velasco E, Eckmanns T. Antibiotics for the common cold: expectations of Germany's general population. Euro Surveill.

2010;15(35): pii=19655. Available online: http://www.eurosurveillance.org/ViewArticle.aspx?Articleld=19655

Article published on 2 September 2010

Physicians mention patients' expectations as a reason for prescribing antibiotics for common (viral) upper respiratory tract infections despite clinical evidence against their use and the physicians' better judgement. We aimed to assess the prevalence of such expectations and factors of influence (knowledge and attitudes) in Germany's general population. In November 2008, 1,778 persons registered with a large market research company were invited to complete an online questionnaire on expectations concerning prescription of antibiotics and on knowledge and attitudes regarding the effectiveness and use of antibiotics for upper respiratory tract infections. A total of 1,076 persons aged $15-78$ years participated (response: $61 \%$ ), of whom $91.8 \%$ reported using antibiotics 'only if absolutely necessary'. Prescription of antibiotics was expected by $113(10.5 \%)$ of the 1,076 respondents for the common cold and by 997 (92.7\%) for pneumonia. In a logistic regression analysis, predictors for expecting a prescription for antibiotics for the common cold included the following opinions: 'common cold or flu can effectively be treated with antibiotics' (prevalence: $37.6 \%$; odds ratio (OR): 9.6 ; $95 \%$ confidence interval ( $\mathrm{Cl}): 3.8$ to 24.3 ) and 'antibiotics should be taken when having a sore throat to prevent more serious illness' (prevalence 8.6\%; OR: 7.6 ; $95 \% \mathrm{Cl}: 3.9$ to 14.5). Among those expecting a prescription $(n=113)$, $80(71 \%)$ reported that they would trust their physician when he or she deems a prescription unnecessary; a further eight ( $7 \%$ ) would be unsatisfied, but would accept the decision. Our results suggest that only a minority expects antibiotics for the treatment of cold symptoms. Physicians should be educated that their decisions not to prescribe antibiotics for the common cold, even when against patients' expectations, are apparently accepted by the majority.

\section{Introduction}

Most respiratory tract infections (e.g. common cold, influenza and sinusitis) are self-limiting and viral in origin. Thus, antibiotics are rarely necessary or effective [1-3]. While overall figures of outpatient antibiotic use in Germany fall within the lower third of those of
European countries [4], 28\% of German respondents in the recently published Eurobarometer on antimicrobial resistance had taken antibiotics in the past year and more than a third had taken them for a viral infection such as a cold or influenza [5]. In a direct observation study conducted in general practices in Germany, $18 \%$ and $64 \%$ of patients with common cold and sinusitis respectively were prescribed antibiotics [6]. These unnecessary prescriptions are thought to largely contribute to the development of antimicrobial resistance and increasing numbers of infections without treatment options [4].

As a reason for these prescriptions against their better judgment, physicians mention pressure exerted by their patients to receive antibiotics even for minor ailments or diseases of viral origin (such as influenza or the common cold) [7]. Doctors feel the need to give in to this pressure due to time constraints or to avoid losing the patient to another practice.

Public knowledge and attitudes concerning antibiotic use and action differ greatly between countries in Europe and between groups of different socio-economic background. People in northern European countries and those with a higher level of education are among the best informed about the effects and sensible use of antibiotics, whereas there are generally higher levels of misconceptions in southern and eastern European countries and among those with a lower level of education. These geographical and socio-economic differences in knowledge and attitudes can in part explain differences in observed use of antibiotics $[5,8]$.

Large campaigns, educating the public about antibiotic action and responsible antibiotic use, have therefore been conducted in various countries including Australia, Belgium, Canada, the United Kingdom and the United States $[9,10]$ as well as at the European level [11], aiming at decreasing unnecessary antibiotic use and thus slowing down the development of antibiotic resistance. 
Little is known about the prevalence of expectations regarding the prescription of antibiotics for upper respiratory tract infections in Germany and possible determinants of these expectations. With this survey, we try to explore knowledge, attitude and expectations of Germany's general public in order to guide decisions on further preventive measures such as public awareness campaigns.

\section{Methods}

Design, sample size, questionnaire design

We conducted a cross-sectional study among a sample of the German general population using an

\section{TABLE 1}

Demographic characteristics of the study participants in $2008(\mathrm{n}=1,076)$ and general public, Germany

\begin{tabular}{|l|c|c|}
\hline Characteristics & $\begin{array}{c}\text { Number } \\
\text { (percentage) of } \\
\text { study participants }\end{array}$ & $\begin{array}{c}\text { Percentage of } \\
\text { general public aged } \\
\geq 15 \text { years }\end{array}$ \\
\hline Male & $589(54 \cdot 7)$ & 48.9 \\
\hline Age (years) & $95(8.8)$ & 7.4 \\
\hline $15-19$ & $213(19.8)$ & 13.8 \\
\hline $20-29$ & $251(23.3)$ & 14.5 \\
\hline $30-39$ & $271(25.2)$ & 19.5 \\
\hline $40-49$ & $164(15.2)$ & 15.7 \\
\hline $50-59$ & $82(7.6)$ & 29.1 \\
\hline$\geq 60$ & & 27.6 \\
\hline Level of school education & $156(14.5)$ & 23.7 \\
\hline Low & $276(25.7)$ & 48.7 \\
\hline Medium & $644(59.9)$ & \\
\hline High & &
\end{tabular}

a Data for 2007 according to the German Federal Statistical Office [14].
Internet-based questionnaire. A sample size of 1,000 was calculated to yield a precision of 3.1 on a confidence level of $95 \%$, which was judged to be sufficient for the purposes of this study.

In total, 1,778 individuals were selected from a panel of approximately 30,000 Internet users, who registered with a large market research company and had given their consent to be contacted for purposes of (market-) research-oriented studies. Upon invitation via email, participants were able to log on to a website and complete the online questionnaire during a 10-day period in November 2008. They received a small remuneration for their efforts in completing the survey.

The questionnaire consisted of closed questions (multiple-choice, Likert scale) on expectations of prescription of antibiotics from physicians and knowledge and attitudes regarding effectiveness of antibiotics and antibiotic use for upper respiratory tract infections. Questions were mostly selected from published studies with similar objectives (e.g. $[8,10,12])$ and slightly rephrased according to the objectives of this study. Detailed demographic data had been recorded at the time of the participant's registration and kept in a separate database with the market research company. The definition of levels of education, as used in this study, was as follows:

- low - maximum of nine years of basic school education;

- medium - 10 years of extended school education;

- high -12 or 13 years of extended school education, including persons who went on to university.

Participants' answers were directly recorded into a database, merged with demographic data and exported

\section{FIGURE 1}

Relative frequency of participants' responses to statements concerning knowledge of antibiotic action and resistance and normal flora, Germany, 2008 ( $n=1,076)$

A cold or the flu can effectively be treated with antibiotics Antibiotics are effective against viruses

Antibiotics are effective against bacteria

Antibiotics also kill bacteria that normally live in the human gut or on the skin

Antibiotic resistant bacteria could infect me or my family

Antibiotic resistance is a problem in German hospitals

Many of the bacteria that live on the skin or in the gut are useful and protect from diseases

If antibiotics are used too often, they are less likely to work in the future

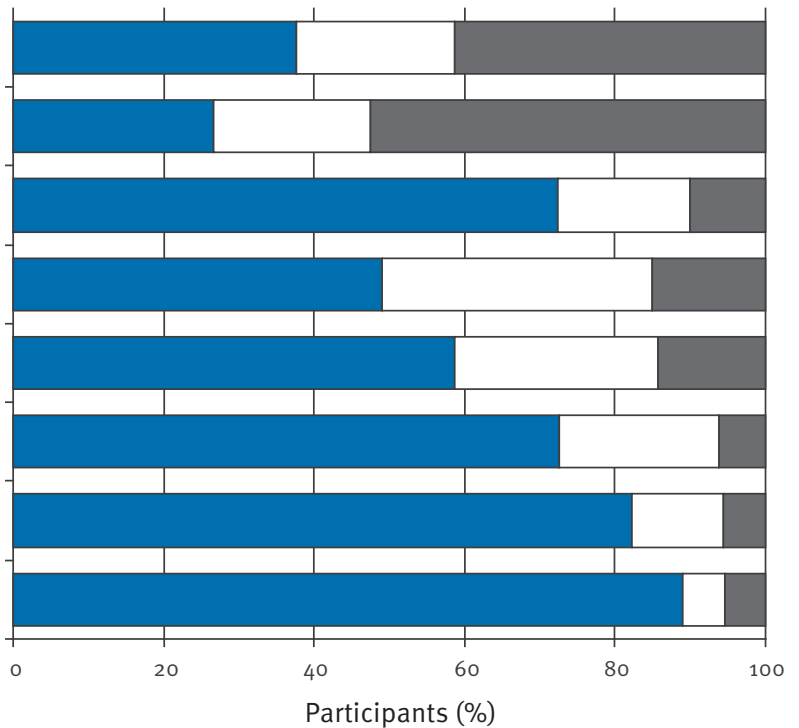


to a single database that was then checked for missing data and monotonous answers (e.g. yes/no only). Variables were dichotomised if needed for the analysis (e.g. 'agree fully' and 'agree somewhat' = 'agree', 'disagree fully' and 'disagree somewhat' = 'disagree').

\section{TABLE 2}

Number of correct responses (to eight knowledge statements) ${ }^{\mathrm{a}}$ and number of responses indicating responsible antibiotics use (to eight attitude statements) ${ }^{\mathrm{b}}$, by participants' demographic characteristics, Germany, $2008(n=1,076)$

\begin{tabular}{|l|c|c|c|c|}
\multirow{2}{*}{ Characteristics } & \multicolumn{2}{c}{ Knowledge } & \multicolumn{2}{c|}{ Attitudes } \\
\cline { 2 - 5 } & Mean & $95 \% \mathrm{Cl}$ & Mean & $95 \% \mathrm{Cl}$ \\
\hline Sex & 5.12 & $4.94-5.29$ & 6.20 & $6.06-6.34$ \\
\hline Male & 5.25 & $5.07-5.44$ & 6.39 & $6.26-6.52$ \\
\hline Female & 4.17 & $3.74-4.59$ & 5.77 & $5.43-6.12$ \\
\hline Age (years) & 4.94 & $4.65-5.23$ & 6.21 & $6.00-6.42$ \\
\hline $15-19$ & 5.35 & $5.11-5.60$ & 6.34 & $6.15-6.54$ \\
\hline $20-29$ & 5.62 & $5.38-5.86$ & 6.50 & $6.32-6.68$ \\
\hline $30-39$ & 5.48 & $5.16-5.79$ & 6.26 & $6.00-6.51$ \\
\hline $40-49$ & 4.35 & $3.85-4.86$ & 6.26 & $5.96-6.55$ \\
\hline $50-59$ & \multicolumn{5}{|l}{} \\
\hline$\geq 60$ & 4.38 & $4.06-4.71$ & 5.88 & $5.59-6.17$ \\
\hline Level of education & 5.00 & $4.73-5.26$ & 6.24 & $6.04-6.43$ \\
\hline Low & 5.45 & $5.29-5.60$ & 6.41 & $6.29-6.52$ \\
\hline Medium & $\mathbf{5 . 1 8}$ & $\mathbf{5 . 0 5 - 5 . 3 0}$ & $\mathbf{6 . 2 9}$ & $\mathbf{6 . 1 9}-6.38$ \\
\hline High & &
\end{tabular}

$\mathrm{Cl}$ : confidence interval.

a See Figure 1.

b See Figure 2.

\section{Statistical analysis}

We calculated relative frequencies of responses (total and stratified by demographic characteristics or particular items in the questionnaire). Scores were calculated for: (i) knowledge of antibiotics and (ii) responsible antibiotic use, summing up the number of correct responses to statements or answers indicating responsible views of antibiotic use, respectively. The chisquare test, t-test or Cuzick's test for trend was applied to test for significant differences between subgroups.

Determinants (demographics, knowledge and attitudes) for expecting a prescription of antibiotics for the common cold were sought using logistic regression analysis. Variables associated with these expectations in the bivariate analysis ( $p<0.2)$ were entered into the model and retained if the adjusted $p$ value was less than 0.1 (stepwise backward elimination). Logistic regression analysis was conducted with a separate set of variables using mean substitution of missing values (separately for the outcomes 'respondent expects antibiotics' versus 'respondent does not expect antibiotics') [13].

\section{All statistics were conducted using STATA 10.1.}

\section{Results}

Of 1,778 invited, 1,076 persons between the age of 15 and 78 years $(54.7 \%$ male) participated, resulting in an overall response of $61 \%$. Compared with Germany's general population, there was no considerable difference in our sample concerning the distribution of persons across Germany's 16 Laender and the size of places of residence (scale of five ranks), but higher

\section{FIGURE 2}

Relative frequency of participants' responses to statements concerning attitudes towards antibiotics and antibiotic use, Germany, $2008(n=1,076)$

I only take antibiotics, if absolutely necessary

I usually know if I need antibiotics before visiting a doctor

If suffering from a cold or flu, it is appropriate to take antibiotics to get through an important event (e.g. marriage, funeral, exams)

When I'm suffering from a cold or the flu, antibiotics help me to get well quicker

If suffering from a cold or flu, it is appropriate to take antibiotics in order to remain active (e.g. job, family, school, university)

When I'm better, I save the remaining antibiotic tablets to use them when I get sick again

Antibiotics should be available without prescription

When I have a sore throat, I should take antibiotics to prevent more seriousillness

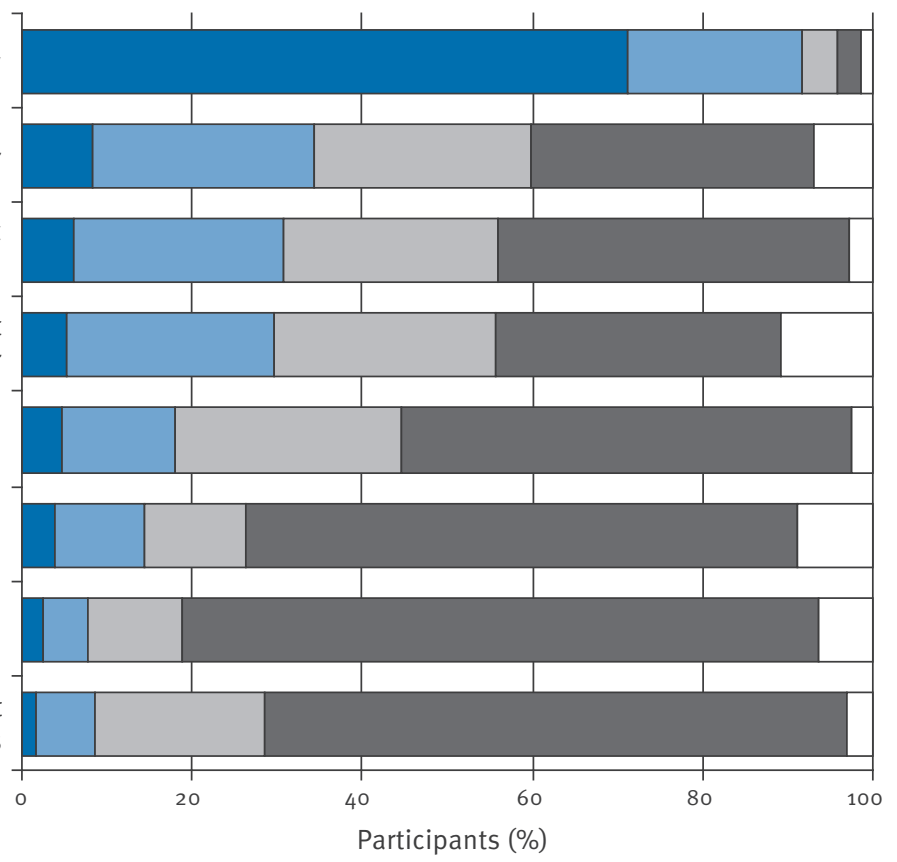


age groups, women and persons with a lower level of education were under-represented (Table 1).

Knowledge and attitudes concerning antibiotics and respiratory tract infections The majority of participants knew that antibiotics are effective against bacteria ( $72.3 \%$ ) but not viruses (52.6\%), knew about antibiotic resistance (89\%) and acknowledged it to be a problem in German hospitals (72.6\%). However, only 445 (41.4\%) knew that antibiotics are not effective against the common cold or influenza (Figure 1).

The mean number of correct responses to eight statements on antibiotic knowledge was 5.2 of eight (65\%). Participants with a high level of education responded to more statements correctly than those with a medium or low level of education (Cuzick's test for trend: p<0.001). Persons of younger (15-29 years) or older ( $\geq 60$ years) age had lower scores in the questions on antibiotic knowledge (Table 2), also after stratification by level of education (data not shown).

When asked about views on antibiotics and antibiotic use, most participants ( $91.8 \%)$ reported that they use antibiotics 'only if absolutely necessary', and disagreed with the statement 'antibiotics should be available without prescription' (86.0\%). However, 34.4\% thought they knew if they needed antibiotics before visiting a doctor and $30.8 \%$ considered it appropriate to take antibiotics to get through an important event when suffering from a cold or influenza (Figure 2). Overall, self-reported views on antibiotics were more sensible or responsible in persons with higher levels of education and least in participants less than 20 years of age (Table 2).

\section{Prevalence of expectations}

Participants were asked on two occasions during the survey whether they expect their physician to prescribe antibiotics for the common cold: the first question dealt with general expectations when consulting their physician because of the common cold or influenza. Most respondents reported that they consult in order to 'be examined, receive advice or a sick certificate' $(47.3 \%)$ or for symptomatic treatment $(44.4 \%)$. A wish for antibiotics was mentioned by $83(7.7 \%)$ respondents. In the second question, participants were asked whether they would expect a prescription of antibiotics for certain common respiratory infections (along with their typical symptoms). In this question, 113 (10.5\%) reported to expect antibiotics for the common cold (sore throat, blocked nose, cough), while $46.9 \%$ and $92.7 \%$ did so for influenza (fever, fatigue, head- and muscle aches, cough) and pneumonia, respectively. For the common cold, the prevalence of self-reported expectations of receiving a prescription of antibiotics depended on level of education in the bivariate analysis $(19.9 \%, 12.0 \%$ and $7.6 \%$ for low, medium and high level of education, respectively, p<0.01). No other significant associations with demographic data (age group, sex, place of residence, migration background, household income, type of health insurance, occupational group) were seen after stratification by level of education (data not shown).

\section{Association of expectations and \\ knowledge and attitudes}

In the multivariable analysis, the strongest predictors for expecting a prescription of antibiotics for the common cold were holding the following opinions: 'a cold or the flu can effectively be treated with antibiotics' (prevalence: $37.6 \%$; odds ratio (OR): 9.6 ; $95 \%$ confidence interval (Cl): 3.8 to 24.3 ) and 'when I have a sore throat, I should take antibiotics to prevent more

\section{TABLE 3}

Multivariable analysis: factors associated with self-reported expectations for antibiotic prescription for the common cold, Germany, $2008(n=1,076)$

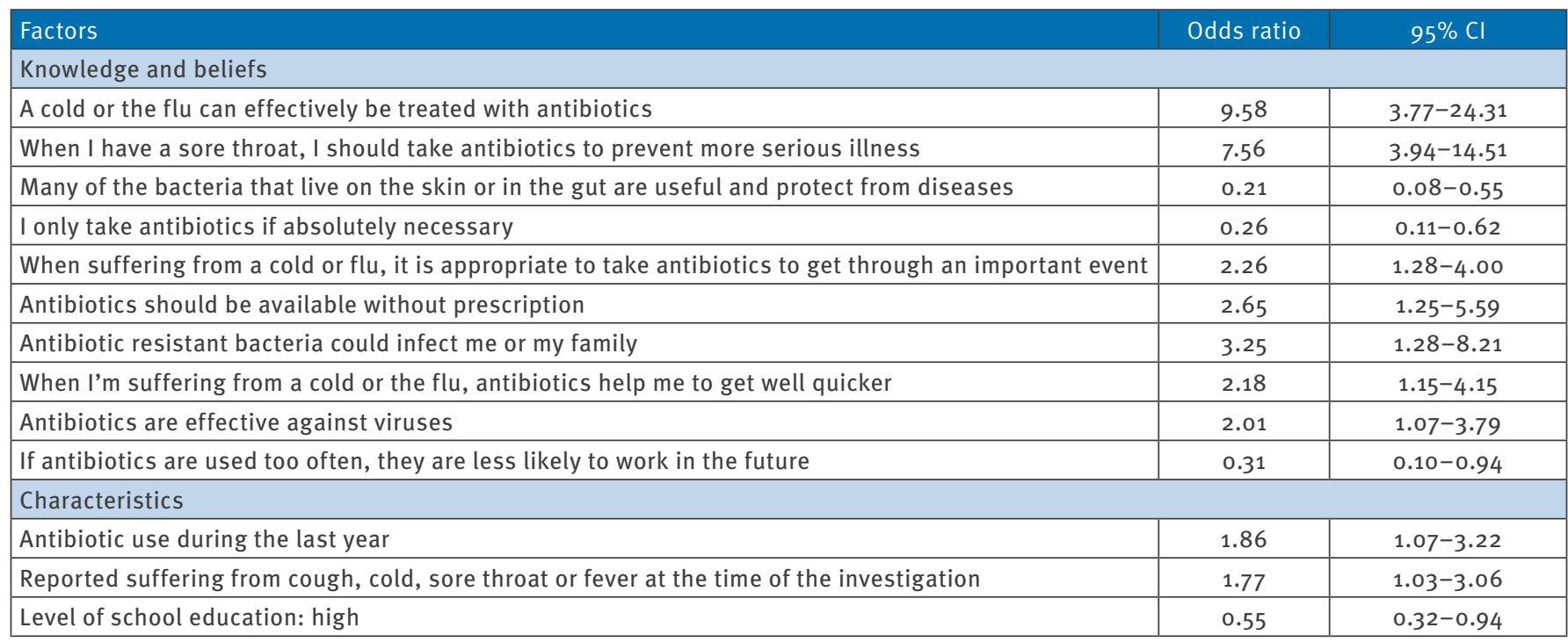


serious illness' (prevalence $8.6 \%$; OR: 7.6 ; $95 \% \mathrm{Cl}: 3.9$ to $14 \cdot 5)$. The full results are shown in Table 3.

\section{Confidence in the physicians' decisions}

Among those expecting a prescription of antibiotics for the common cold $(n=113), 80(71 \%)$ reported that they trust their physician when he or she deems a prescription unnecessary. A further eight ( $7 \%$ ) would be unsatisfied but accept the decision, whereas 14 (12\%) reported that they would win over the doctor to prescribe and three (3\%) would consult another doctor. In a more general question, 99 of 1076 (9.2\%) reported that they felt they were not taken seriously or were not receiving proper treatment if they were not prescribed antibiotics for a cold or influenza.

\section{Discussion}

We found that $10.5 \%$ of respondents expected a prescription of antibiotics for the common cold and that such expectation was associated with a lack of knowledge of correct indications for antibiotic use and antibiotic resistance. Of those expecting antibiotics from a consultation, $\mathbf{7 7 . 9 \%}$ reported that they trusted their physician when he or she deemed a prescription unnecessary or would at least accept such a decision.

\section{Strengths and limitations}

This is, to our knowledge, the largest study specifically investigating public views and knowledge of common respiratory tract infections and antibiotic use in Germany. The use of an online access panel allowed us to achieve a high response and to gain insight into the views and expectations of a wide range of population groups before they visit a doctor. Our study has limitations: firstly, the shortage of participants with a lower education level might bias the overall results towards better knowledge and more responsible views than actually present in the general population. We therefore presented stratified results whenever appropriate. Secondly, asking the general public might introduce a bias towards a lower prevalence of expectations of receiving a prescription of antibiotics when compared with asking patients. We therefore included a question on the presence of common cold or influenza symptoms at the time of investigation, which allowed us to partially compensate for this effect. Finally, as with all questionnaire studies, participants may give answers that they consider are socially desirable, which might introduce a bias towards more responsible use of antibiotics.

\section{Patients' expectations}

The existence of patients' expectations regarding the prescription of antibiotics and their influence on the decisions of doctors to prescribe is unequivocal $[7,15,16]$; however, the prevalence of such expectations varies considerably depending on the setting or type of study. It can be as high as 50\% in United States adults consulting for cold symptoms [17] or as low as $1.2 \%$ in the Dutch general population [12].
Overall, our results indicate a sensible approach to antibiotics among Germany's public. Only a minority reported that they expected a prescription of antibiotics for cold symptoms and most reported to be taking antibiotics 'only if absolutely necessary'.

This is remarkable in light of the overprescription of antibiotics and the common belief that patient expectations at least partly drive it. However, our findings are in line with several studies that show that most patients seek information, reassurance or a diagnosis rather than a prescription of any kind [18] or a prescription of antibiotics in particular $[19,20]$. Real expectations of patients regarding the prescription of medication seem to be much less prevalent than expectations perceived by the doctor and furthermore their presence less predictive of the decision to prescribe [21-23]. Cockburn et al. found that when a patient expected a prescription he was three times more likely to receive it, but when the general practitioner thought the patient expected medication, the patient was 10 times more likely to receive it [22]. A study conducted in general practices in Germany showed that nearly all patients who, in their doctor's opinion, expected a drug left the surgery with a prescription. However, doctors accurately perceived the patient's wish for a drug prescription in only $41 \%$ of cases [24].

Furthermore, if patients do expect a prescription for cold symptoms, they do not necessarily expect a prescription of antibiotics. Van Driel et al. suggested that patients with acute sore throat and who hope for antibiotics are actually seeking treatment for pain [19]. This corresponds well to results of our survey, where $44 \%$ of respondents reported to expect symptomatic treatment for cold symptoms (e.g. lozenges, painkiller, cough medication) while only $7.7 \%$ reported to expect antibiotics for these symptoms.

In contrast to the observed low prevalence of expecting antibiotics for the common cold, nearly half of the participants in our study reported to expect a prescription of antibiotics for influenza. Given the existence of antiviral medication used for the treatment of influenza, it is unclear whether this question was not specific enough or whether influenza is much more frequently expected to be treated with antibiotics. But even if an individual patient has such expectations and the physician denies an actual wish for a prescription of antibiotics, he or she must not necessarily worry about losing the patient to another practice. The results of our study indicate a high level of confidence towards physicians and their decisions among Germany's general public. Less than $3 \%$ of those reporting to expect an antibiotic for cold symptoms stated that they would consult another doctor if their request were denied. Studies conducted in general practice settings showed similar results and concluded that a medically justified refusal to prescribe antibiotics had, in most cases, no negative effect on the consultation or its assessment by the patient $[15,24,25]$. 


\section{Misconceptions and their implications}

Misconceptions concerning the appropriateness and effectiveness of antibiotics for different indications seem to be quite common among Germany's public, comparable with results found in similar studies conducted in the United Kingdom [10] or the Netherlands [12]. In our multivariable analysis, these misconceptions were clearly associated with the expectation of receiving antibiotics for the common cold. The two 'items' most strongly associated were both related to the plain beliefs that antibiotics can be used to effectively treat the common cold or influenza and if they are used for a sore throat they could prevent more serious illness. It therefore seems to make sense to educate the public on antibiotic effectiveness, correct indications and risks of antibiotic mis- or overuse. If this is considered, efforts should be focused on the group where relevant expectations are most prevalent: those with a lower level of education. However, simply educating the public may not be effective in reducing the level of prescribing. A large household survey conducted in the United Kingdom demonstrated that those with a greater knowledge of antibiotics were no less likely to be prescribed an antibiotic [10]. According to a systematic review of 39 studies focusing on interventions to improve antibiotic prescription practices in ambulatory care, multifaceted interventions involving informing patients, communication training of physicians and educating the public were more successful [26]. In a cluster-randomised study conducted in more than 100 general practices in Germany, an intervention focusing on doctor-patient communication and patient empowerment even reduced antibiotic prescription rates for acute cough by $40 \%$ after 12 months [23].

\section{Conclusions and recommendations}

Our study suggests that there may be several opportunities to reduce unwarranted use of antibiotics and thus ultimately reduce further development of antibiotic resistance. Expectations that antibiotics will be prescribed for the common cold are generally not widespread and are most likely less prevalent than believed by general practitioners.

Physicians should therefore carefully explore if a perceived wish for antibiotics really exists in an individual patient. It may turn out that the consulting patient actually seeks symptomatic relief, reassurance or just a sick certificate.

Existing erroneous expectations might be caused by misconceptions of what can be achieved by taking antibiotics for cold symptoms and what risks are involved (e.g. adverse effects or development of resistance). With the high level of confidence physicians enjoy among the public, they may often be able to convince patients of alternative strategies and should not overly worry that they may displease their patients by not yielding to their requests. Change, however, does not come easily and multifaceted approaches are needed to tackle the problem of overprescribing and antibiotic resistance.
Acknowledgements

This work was supported by the Robert Koch Institute, Berlin, Germany. The cost of the survey and remuneration of participants was covered by the market research company.

\section{References}

1. Williamson IG, Rumsby K, Benge S, Moore M, Smith PW, Cross $M$, et al. Antibiotics and topical nasal steroid for treatment of acute maxillary sinusitis: a randomized controlled trial. JAMA. 2007;298(21):2487-96.

2. Little P, Williamson I, Warner G, Gould C, Gantley M, Kinmonth $A L$. Open randomised trial of prescribing strategies in managing sore throat. BMJ. 1997;314(7082):722-7.

3. Young J, De Sutter A, Merenstein D, van Essen GA, Kaise $\mathrm{L}$, Varonen $\mathrm{H}$, et al. Antibiotics for adults with clinically diagnosed acute rhinosinusitis: a meta-analysis of individual patient data. Lancet. 2008;371(9616):908-14.

4. Goossens H, Ferech M, Vander Stichele R, Elseviers M, ESAC Project Group. Outpatient antibiotic use in Europe and association with resistance: a cross-national database study. Lancet. 2005;365(9459):579-87.

5. European Commission. Antimicrobial resistance. Special Eurobarometer 338. April 2010. Report. Available from: http://ec.europa.eu/health/antimicrobial_resistance/docs/ ebs_338_en.pdf

6. Fischer T, Fischer S, Kochen MM, Hummers-Pradier E. Influence of patient symptoms and physical findings on general practitioners' treatment of respiratory tract infections: a direct observation study. BMC Fam Pract. 2005;6(1):6.

7. Barden LS, Dowell SF, Schwartz B, Lackey C. Current attitudes regarding use of antimicrobial agents: results from physician's and parents' focus group discussions. Clin Pediatr (Phila). 1998;37(11):665-71.

8. Grigoryan L, Burgerhof JG, Degener JE, Deschepper R, Lundborg CS, Monnet DL, et al. Attitudes, beliefs and knowledge concerning antibiotic use and self-medication: a comparative European study. Pharmacoepidemiol Drug Saf. 2007;16(11):1234-43.

9. Finch RG, Metlay JP, Davey PG, Baker LJ, International Forum on Antibiotic Resistance colloquium. Educational interventions to improve antibiotic use in the community: report from the International Forum on Antibiotic Resistance (IFAR) colloquium, 2002. Lancet Infect Dis. 2004;4(1):44-53.

10. McNulty CA, Boyle P, Nichols T, Clappison P, Davey P. Don't wear me out--the public's knowledge of and attitudes to antibiotic use. J Antimicrob Chemother. 2007;59(4):727-38.

11. European Antibiotic Awareness Day. A European Health Initiative. Stockholm: European Centre for Disease Prevention and Control. [Accessed 1 Apr 2010]. Available from: http://ecdc. europa.eu/en/eaad/Pages/Home.aspx

12. Cals JW, Boumans D, Lardinois RJ, Gonzales R, Hopstaken RM, Butler CC, et al. Public beliefs on antibiotics and respiratory tract infections: an internet-based questionnaire study. $\mathrm{Br}$ J Gen Pract. 2007;57(545):942-7.

13. Yuen Fung K, Wrobel BA. The treatment of missing values in logistic regression. Biometrical Journal. 1989;31(1):35-47.

14. Federal Statistical Office Germany. Genesis-online. [Accessed 18 Dec 2008]. German. Available from: https://www-genesis. destatis.de/genesis/online

15. Butler CC, Rollnick S, Pill R, Maggs-Rapport F, Stott N. Understanding the culture of prescribing: qualitative study of general practitioners' and patients' perceptions of antibiotics for sore throats. BMJ. 1998;317(7159):637-42.

16. Macfarlane J, Holmes W, Macfarlane R, Britten N. Influence of patients' expectations on antibiotic management of acute lower respiratory tract illness in general practice: questionnaire study. BMJ. 1997;315(7117):1211-4.

17. Braun BL, Fowles JB. Characteristics and experiences of parents and adults who want antibiotics for cold symptoms. Arch Fam Med. 2000;9(7):589-95.

18. Ruiz-Moral R, Perula de Torres LA, Jaramillo-Martin I. The effect of patients' met expectations on consultation outcomes. A study with family medicine residents. J Gen Intern Med. 2007;22(1):86-91.

19. van Driel ML, De Sutter A, Deveugele M, Peersman W, Butler CC, De Meyere M, et al. Are sore throat patients who hope for antibiotics actually asking for pain relief? Ann Fam Med. 2006;4(6):494-9. 
20. Welschen I, Kuyvenhoven M, Hoes A, Verheij T. Antibiotics for acute respiratory tract symptoms: patients' expectations, GPs' management and patient satisfaction. Fam Pract. 2004;21(3):234-7.

21. Britten $N$, Ukoumunne 0 . The influence of patients' hopes of receiving a prescription on doctors' perceptions and the decision to prescribe: a questionnaire survey. BMJ. 1997;315(7121):1506-10.

22. Cockburn J, Pit S. Prescribing behaviour in clinical practice: patients' expectations and doctors' perceptions of patients' expectations--a questionnaire study. BMJ. 1997;315(7107):520-3.

23. Altiner A, Brockmann S, Sielk M, Wilm S, Wegscheider K, Abholz HH. Reducing antibiotic prescriptions for acute cough by motivating GPs to change their attitudes to communication and empowering patients: a cluster-randomized intervention study. J Antimicrob Chemother. 2007;60(3):638-44.

24. Himmel W, Lippert-Urbanke E, Kochen MM. Are patients more satisfied when they receive a prescription? The effect of patient expectations in general practice. Scand J Prim Health Care. 1997;15(3):118-22.

25. Hamm RM, Hicks RJ, Bemben DA. Antibiotics and respiratory infections: are patients more satisfied when expectations are met? J Fam Pract. 1996;43(1):56-62.

26. Arnold SR, Straus SE. Interventions to improve antibiotic prescribing practices in ambulatory care. Cochrane Database Syst Rev. 2005;(4):CDo03539. 